\title{
Beneficial effects of exercise: shifting the focus from body weight to other markers of health*
}

\author{
N A King, ${ }^{1}$ M Hopkins, ${ }^{2}$ P Caudwell, ${ }^{3}$ R J Stubbs, ${ }^{4}$ J E Blundell ${ }^{3}$ \\ ${ }^{1}$ Institute of Health and Biomedical Innovation, School of Human Movement Studies, Queensland University of Technology, Brisbane, Aus- \\ tralia \\ ${ }^{2}$ Department of Health and Exercise Science, Trinity and All Saints College, Leeds, UK \\ ${ }^{3}$ Biopsychology Group, Institute of Psychological Sciences, University of Leeds, Leeds, UK \\ ${ }^{4}$ Slimming World, Clover Nook Road, Somercotes, Alfreton, UK
}

\begin{abstract}
Background: Exercise is widely promoted as a method of weight management, while the other health benefits are often ignored. The purpose of this study was to examine whether exercise-induced improvements in health are influenced by changes in body weight.

Methods: Fifty-eight sedentary overweight/obese men and women (BMI 31.8 (SD 4.5) $\mathrm{kg} / \mathrm{m}^{2}$ ) participated in a 12-week supervised aerobic exercise intervention $(70 \%$ heart rate max, five times a week, $500 \mathrm{kcal}$ per session). Body composition, anthropometric parameters, aerobic capacity, blood pressure and acute psychological response to exercise were measured at weeks 0 and 12.

Results: The mean reduction in body weight was $-3.3(3.63) \mathrm{kg}$ $(p<0.01)$. However, 26 of the 58 participants failed to attain the predicted weight loss estimated from individuals' exercise-induced energy expenditure. Their mean weight loss was only -0.9 $(1.8) \mathrm{kg}(p<0.01)$. Despite attaining a lower-than-predicted weight reduction, these individuals experienced significant increases in aerobic capacity $(6.3(6.0) \mathrm{ml} / \mathrm{kg} / \mathrm{min} ; p<0.01)$, and a decreased systolic $(-6.00(11.5) \mathrm{mmHg} ; p<0.05)$ and diastolic blood pressure $(-3.9$ (5.8) $\mathrm{mmHg} ; p<0.01)$, waist circumference $(-3.7(2.7) \mathrm{cm}$; $p<0.01)$ and resting heart rate $(-4.8(8.9) \mathrm{bpm}, p<0.001)$. In addition, these individuals experienced an acute exercise-induced increase in positive mood.

Conclusions: These data demonstrate that significant and meaningful health benefits can be achieved even in the presence
\end{abstract}

\section{Correspondence to:}

Dr N King, Institute of Health and Biomedical Innovation, School of Human Movement Studies, Queensland University of Technology, Brisbane, 4059, QLD, Australia; n.king@qut.edu.au

Parts of these data were presented at BASES in UK, 2006, and at the 9th ICO in Australia, 2006. Accepted 24 August 2009 Published Online First 29 September 2009

*This article has been reproduced with permission from the BMJ Group. It was originally published in the British Journal of Sports Medicine 2009;43:924-927. of lower-than-expected exercise-induced weight loss. A less successful reduction in body weight does not undermine the beneficial effects of aerobic exercise. From a public health perspective, exercise should be encouraged and the emphasis on weight loss reduced.

It is difficult to ignore the media attention and public health messages about the current obesity epidemic and the emphasis to reduce body weight. Despite some cynicism about the true status and implications of the obesity epidemic, ${ }^{1}$ there is no doubt that preventing weight gain can contribute to improving the health of the nation. ${ }^{2}$ The efficacy of exercise as a means of weight reduction is regularly scrutinised and doubted. ${ }^{3}$ Body weight loss is commonly regarded as the marker of efficacy by researchers, and more typically the perceived measure of success by lay people. Any lack of weight loss associated with exercise is often attributed to poor compliance and/or compensation for the acute exercise-induced increase in energy expenditure. ${ }^{4}$ That is, the net change in exercise-induced energy expenditure is modest and insignificant due to compensatory adjustments in energy intake and a failure to comply fully with the exercise prescription. Clearly, if people do not comply with the exercise prescribed (by themselves or others), the expected weight loss will not occur. The success of exercise in promoting weight loss will vary between individuals; ${ }^{5}$ however, those who lose less weight should not be labelled as failures or be perceived negatively. Evidence suggests that individuals have unrealistic weight loss expectations, ${ }^{6}$ which is indicative of an inappropriate focus on body weight. Blair and Lamonte suggested that 'a focus on weight loss is often counterproductive and unsuccessful, and sometimes may even be unnecessary. ${ }^{7}$ Furthermore, body weight per se might not be the most important risk factor for obesity comorbidities. ${ }^{8-10}$ It is possible that media attention and the persistent barrage of messages to reduce obesity are to blame for the obsession with the capacity of exercise to produce marked and rapid weight loss. Exercise gives rise to a wide range of health benefits, not just weight loss. ${ }^{11}$

Disappointment and low self-esteem associated with poor weight loss could lead to low exercise adherence and a general perception that exercise is futile and not beneficial. This viewpoint is potentially damaging - a more transparent and positive attitude to the health benefits of exercise is required. Individuals who drop out of exercise interventions - possibly due to disappointing weight loss - have a history of previous weight loss attempts, and exercise adherence is associated with intrinsic motivation. ${ }^{12,13}$ Unfortunately, focusing on 
exercise-induced changes in body weight undermines the arguably more important health benefits associated with exercise. Although there is some debate about the direct association between weight loss per se and health benefits, there is evidence to suggest that reductions of $5-10 \%$ in body weight improve some health risk markers. ${ }^{14}$ For several years Steve Blair has promoted the idea that fitness is more important than fatness, since there are data to demonstrate that a fat but fit person has fewer health risks than a lean but unfit individual. ${ }^{15}$ There is a real need to promote physical activity and to prevent it being undervalued by the community and by public health professionals. ${ }^{16}$

In fact, when sedentary people undertake exercise, the activity provides a massive stimulus with widespread physiological implications. The effect cannot be readily anticipated, but studies have noted considerable diversity in the responses. ${ }^{17,18}$ In addition, the energy expended in exercise is believed, by some, ${ }^{19}$ to stimulate compensation so that energy balance is preserved. We have examined these issues under controlled scientific conditions.

\section{MATERIALS AND METHODS}

Fifty-eight sedentary overweight/obese men (BMI 30.5 (SD 3.3) kg/ $\mathrm{m}^{2}$ ) and women (BMI $32.6(4.8) \mathrm{kg} / \mathrm{m}^{2}$ ) completed a 12-week supervised aerobic exercise programme ( $70 \%$ heart rate max) five times a week in the Human Appetite Research Unit at the University of Leeds. Each exercise session was designed to expend approximately 500 kcal. Body composition (air plethysmography - Bodpod, Concord), anthropometry, aerobic capacity (submaximal $\mathrm{VO}_{2}$ max test), blood pressure, resting heart rate and the acute affective response (Positive and Negative Affect Scale (PANAS) ${ }^{20}$ ) to exercise were measured at weeks 0 and 12. Subjects were instructed not to restrict their energy intake during the study.

\section{RESULTS}

When all 58 subjects' data were pooled, the mean reduction in body weight was $-3.3(3.6) \mathrm{kg}(p<0.01)$. However, there was large interindividual variability (Fig. 1). Based on empirical evidence, ${ }^{21}$ the mean weight loss matched the predicted weight loss. Further examination of the weight-change data revealed that subjects could be categorised into two groups (responders and non-responders) based solely on their actual initial weight relative to the calculated weight change. Calculations were based on the assumed energy costs of $9540 \mathrm{kcal} /$ $\mathrm{kg}$ and $1100 \mathrm{kcal} / \mathrm{kg}$ of fat mass and fat-free mass respectively. ${ }^{21}$

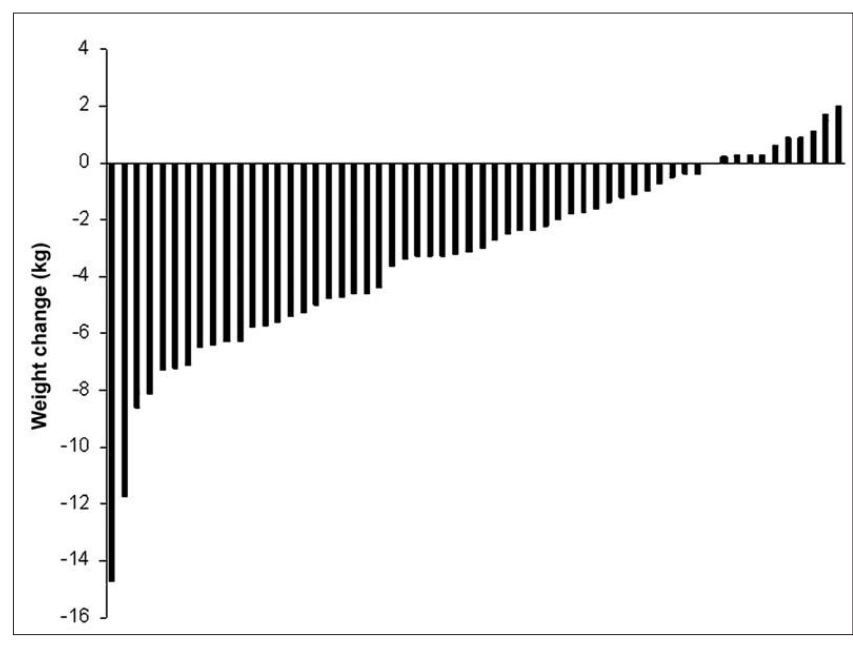

Fig. 1. Variability in individual changes in body weight (kg) after 12 weeks of monitored exercise.

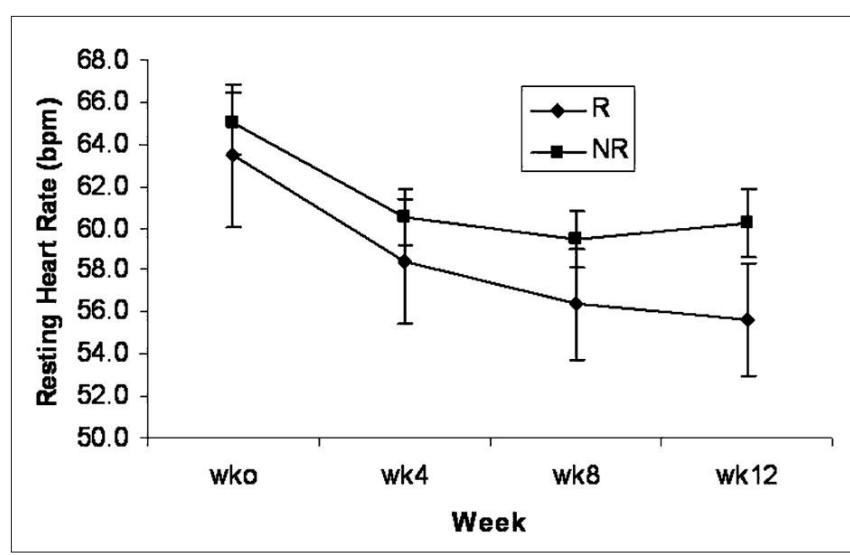

Fig. 2. Mean reduction in resting heart rate during the 12-week exercise intervention in $R$ and NR. There was a significant main effect of week but no statistically significant main effect of group or group $X$ time interaction.

The non-responders $(n=26)$ lost less weight than predicted based on their individual total exercise-induced energy expenditure. Although statistically significantly lower than baseline (week 0 ), their mean weight loss was only $-0.9(1.8) \mathrm{kg}(p<0.01)$, compared with the remaining participants (responders) who experienced an average weight loss of $-5.2(3.64) \mathrm{kg}(p<0.01)$. Indeed, some of the classified non-responders actually gained weight. Therefore, based on body weight alone, exercise could be regarded as ineffective and futile for the non-responders (and even counterproductive for the weight gainers). However, the effectiveness of exercise should not be exclusively judged on changes in body weight because it undermines the other health benefits that are commonly associated with exercise. Despite the lower-than-expected weight loss, the non-responders did achieve improvements in health markers. They experienced a significant increase in aerobic capacity $(6.3(6.0) \mathrm{ml} / \mathrm{kg} / \mathrm{min} ; p<0.01)$, reduction in waist circumference $(-3.08(2.66) \mathrm{cm} ; p<0.01)$, and decreases in systolic $(-6.0(11.5) \mathrm{mmHg} ; p<0.05)$, diastolic blood pressure $(-3.9(5.8) \mathrm{mmHg} ; p<0.01)$ and resting heart rate $(-4.8(8.9)$ bpm; $p<0.001)$ (Fig. 2). The reduction in both systolic and diastolic blood pressure was more marked when examining the changes in those individuals who were classed as hypertensive $(140 / 90 \mathrm{mmHg})$ at baseline. They experienced a significant reduction in systolic $(-15$ (10.4) $\mathrm{mmHg} ; p<0.0001)$ and diastolic $(-10(4.6), p<0.0001)$ blood pressure.

In addition to the reduction in health risk markers, the nonresponders experienced an acute improvement in psychological state reflected in the exercise-induced increase in positive mood, which was maintained during the 12 weeks. Interestingly, although the difference in weight loss between the groups was statistically significant, there were no statistically significant differences in the health benefits. Furthermore, when all subjects' data were pooled, there was no association between weight loss and improvements in health markers. Therefore, the exercise itself, independent of weight loss, made a significant contribution to the health benefits. There were no significant differences between men and women, and the proportion of men in each group was similar (responders=men:women, 9:23, $28 \%$ and non-responders=men:women, 10:16, 38\%). Moreover, these results show that if people fail to lose weight following a recommendation to perform physical activity, it is not necessarily a result of poor compliance. In some individuals who are resistant to weight loss, it will be due to strong physiological compensatory processes. 


\begin{tabular}{|c|c|c|c|}
\hline Variable & Group & Absolute change & Change (\%) \\
\hline \multirow[t]{2}{*}{ Body mass (kg) } & Responders & -5.2 & -5.7 \\
\hline & Non-responders & -0.9 & -1.0 \\
\hline \multirow[t]{2}{*}{$\mathrm{BMI}\left(\mathrm{kg} / \mathrm{m}^{2}\right)$} & Responders & -1.8 & -5.7 \\
\hline & Non-responders & -0.3 & -1.0 \\
\hline \multirow[t]{2}{*}{ Fat mass (kg) } & Responders & -4.9 & -15.3 \\
\hline & Non-responders & -1.2 & -4.7 \\
\hline \multirow[t]{2}{*}{ Body fat (\%) } & Responders & -3.5 & -10.5 \\
\hline & Non-responders & -1.1 & -3.7 \\
\hline \multirow[t]{2}{*}{ Lean mass (kg) } & Responders & -0.3 & -0.6 \\
\hline & Non-responders & +0.3 & +0.4 \\
\hline \multirow[t]{2}{*}{ Waist circumference (cm) } & Responders & -6.0 & -5.8 \\
\hline & Non-responders & -3.7 & -3.7 \\
\hline \multirow[t]{2}{*}{$\mathrm{VO}_{2} \max (\mathrm{ml} / \mathrm{kg} / \mathrm{min})$} & Responders & +9.1 & +32.5 \\
\hline & Non-responders & +6.3 & +23.0 \\
\hline \multirow[t]{2}{*}{ Diastolic blood pressure $(\mathrm{mmHg})$} & Responders & -3.4 & -3.7 \\
\hline & Non-responders & -3.9 & -4.6 \\
\hline \multirow[t]{2}{*}{ Systolic blood pressure (mmHg) } & Responders & -2.9 & -1.9 \\
\hline & Non-responders & -6.0 & -4.3 \\
\hline
\end{tabular}

\section{DISCUSSION}

These data demonstrate that significant and meaningful health benefits can be achieved even in the presence of lower-than-expected exercise-induced weight loss. A novel feature of this study is that the exercise intervention was supervised, and each session was monitored and measured directly. Therefore, unlike most of the other exercise intervention trials, we can guarantee that the lowerthan-expected weight loss was not due to poor compliance. Indeed, the degree of adherence and total exercise energy expenditures did not differ between the two groups.

Importantly, based on each individual's predicted weight loss, those who lost less weight than predicted still experienced improvements in other markers of health. It is important to note that these health markers are not overtly accessible to most individuals, whereas other markers such as body weight, perceptions of fitting of clothes and perceived body image are more accessible and transparent. Therefore, most individuals are 'blind' to the exerciseinduced health improvements. Indeed, these data demonstrate that subjects who lost less than the predicted weight still experienced a mean reduction of approximately $3.7 \mathrm{~cm}$ in waist circumference. Waist circumference is promoted as being more important than BMI at predicting risk of obesity-related disease, ${ }^{22,23}$ and a better marker of success than BMI in response to exercise. ${ }^{24}$ The reductions in diastolic and systolic blood pressure in the non-responders were similar to other studies. ${ }^{25}$

There is a need to increase knowledge and understanding of the health benefits of exercise, and reduce the emphasis on weight loss. ${ }^{11}$ This agrees with the evidence that cardiorespiratory fitness is a more powerful predictor of risk than body weight. ${ }^{16}$ In addition, evidence from the Finnish Diabetes Preventions study showed that individuals who did not lose weight but who did increase their physical activity maintained a reduction in the risk of diabetes. ${ }^{26}$ Exercise should be promoted as an optimistic method of improving weight management and overall health by highlighting the importance of using other markers of success. Weight loss is not the only benefit of exercise; nor is it the most useful and appropriate marker of health. ${ }^{27}$ A recent intervention in postmenopausal obese women using low-intensity, low-volume exercise showed improvements in cardiorespiratory fitness with no effect on body weight. ${ }^{28}$ Furthermore, partly due to the culture of focusing on obesity and weight loss, individuals will actively seek opportunities which are specifically targeted to promote weight loss - and exercise is one of those. From a public health perspective, exercise should therefore be encouraged; and even though body weight may not change markedly, or match expectations, lean tissue will be increased (or preserved), and body shape will change (waist circumference). There will also be a lowering of risk factors for comorbidity problems and diabetes. In the present study, the reduction in waist circumference - even in the non-responders - is important, since this variable is a proxy measure of visceral fat which is highly associated with cardiometabolic risk factors. ${ }^{11}$ It may therefore be important to encourage the replacement of BMI and body weight with waist circumference as a measure of the effectiveness of exercise.

Within this framework, there is a key role for physicians and health professionals. These professionals (including dietitians) not only can promote physical activity as a contribution to health but also can be instrumental in improving weight management in nonresponders (Table I). We have demonstrated that non-responders fail to lose the expected weight because of an increase in appetite reflected in an increased selection of high-fat foods and a decrease in fruit and vegetable consumption ${ }^{29}$ and because of an increased orexigenic (hunger) response. ${ }^{30}$ Therefore, using dietary behaviour 
strategies, dietitians and health professionals could help to counter appetite stimulation in the non-responders and therefore help weight management while preserving all of the health benefits of exercise.

In conclusion, these data provide support for the belief that poor weight loss associated with exercise should not undermine its capacity to improve health. Health professionals, it can be argued, have a responsibility to promote exercise, publicise the health benefits independent of body weight and, more importantly, shift the focus from changes in body weight to changes in overall physical and psychological well-being. Our intervention study has clearly demonstrated that when exercise is carried out, people experience beneficial physiological and psychological effects independent of any effect on body weight.

However, the implication of these results for weight management and the obesity epidemic should be interpreted carefully. The results do not mean the exercise is fruitless or ineffective in the battle against obesity. Overall, exercise can help to check weight gain, and in some people it is very effective. Others need additional help to deal with any compensatory response.

Funding: This study forms part of a larger project funded by the Biotechnology and Biological Sciences Research Council (BBS/ B/05079).

Competing interests: None.

Ethics approval: Ethics approval was provided by University of Leeds Ethics Committee.

Patient consent: Obtained.

Provenance and peer review: Not commissioned; not externally peer reviewed.

\section{REFERENCES}

1. Campos P, Saguy A, Ernsberger E, et al. The epidemiology of overweight and obesity: public health crisis or moral panic? Int J Epidemio 2005;35:55-60.

2. Rigby N. Commentary: Counterpoint to Campos et al. Int J Epidemio 2005;35:79-80.

3. Garrow JS, Summerbell CD. Meta-analysis: effect of exercise, with or without dieting, on the body composition of overweight subjects. Eur J Clin Nutr 1995;49:1-10.

4. King NA, Hopkins M, Caudwell P, et al. Involuntary and voluntary compensatory responses to exercise interventions: barriers to weight loss. Obesity 2007;15:1373-83.

5. King NA, Caudwell P, Hopkins $M$, et al. Individual variability following 12 weeks of supervised exercise: identification and characterisation of compensation for exerciseinduced weight loss. Int J Obes Rel Met Dis 2007;32:177-84.

6. Foster GD, Wadden TA, Vogt RA, et al. What is a reasonable weight loss? Patients expectations and evaluations of obesity treatment outcomes. J Con Clin Psych 1997;65:79-85.

7. Blair SN, Lamonte MJ. Commentary: current perspectives on obesity and health: black and white, or shades of grey? Int J Epidemiol 2005;35:6972 .

8. Janssen I, Katzmarzyk PT, Ross R. Waist circumference and not body mass index explains obesity-related health risk. Am J Clin Nutr 2004;79:379-84.

9. Poirier $P$, Lemieux I, Mauriege $P$, et al. Impact of waist circumference on the relationship between blood pressure and insulin: the Quebec Health Survey. Hypertension 2005;45:363-7.
10. Janiszewski PM, Janssen I, Ross R. Does waist circumference predict diabetes and cardiovascular disease beyond commonly evaluated cardiometabolic risk factors? Diab Car 2007;30:3105-9.

11. Ross R, Janiszewski. Is weight loss the optimal target for obesity-related cardiovascular disease risk reduction? Can J Cardiol 2008;24:25D-31D.

12. Teixeira PJ, Going SB, Sardinha LB, et al. A review of psychosocial pretreatment predictors of weight control. Obes Rev 2005;6:43-65.

13. Teixeira PJ, Going SB, Houtkooper LB, et al. Exercise motivation, eating, and body image variables as predictors of weight control. Med Sci Sports Exerc 2006;38:179-88.

14. Van Gaal LF, Wauters MA, De Leeuw IH. The beneficial effects of modest weight loss on cardiovascular risk factors. Int $\mathrm{J}$ Obes Rel Met Dis 1997;21:S5-S9.

15. Blair SN, Kohl HW 3rd, Barlow CE, et al. Changes in physical fitness and all-cause mortality. A prospective study of healthy and unhealthy men. JAMA 1995;273:1093-8.

16. Blair SN. Physical inactivity: the biggest public health problem of the $21 \mathrm{st}$ century. Br J Sprt Med 2009;43:1-2.

17. Snyder KA, Donnelly JE, Jabobsen DJ, et al. The effects of long-term, moderate intensity, intermittent exercise on aerobic capacity, body composition, blood lipids, insulin and glucose in overweight females. Int $J$ Obes Relat Metab Disord 1997;21:1180-9.

18. Donnelly JE, Smith BK. Is exercise effective for weight loss with ad libitum diet? Energy balance, compensation, and gender differences. Exerc Sport Sci Rev 2005;33:169-74.

19. Mayer J, Roy P, Mitra KP. Relation between caloric intake, body weight, and physical work: studies in an industrial male population in West Bengal. Am J Clin Nutr 1956;4:169-74.

20. Watson D, Clark LA, Tellegen A. Development and validation of brie measures of positive and negative affect: The PANAS scales. J Person Soc Psychol 1998;54:1063-70.

21. Leibel RL, Rosenbaum M, Hirsch J. Changes in energy expenditure resulting from altered body weight. N Engl J Med 1995;332:621-8.

22. Katzmarzyk PT, Janssen I, Ross R, et al. The importance of waist circumference in the definition of metabolic syndrome: prospective analyses of mortality in men. Diab Car 2006;29:404-9.

23. Klein S, Allison DB, Heymsfield SB, et al. Waist circumference and cardiometabolic risk: a consensus statement from shaping America's health: Association for Weight Management and Obesity Prevention; NAASO the Obesity Society; the America Society for Nutrition; and the American Diabetes. Diab Care 2007;30:1647-52.

24. Dekker MJ, Lee $S$, Hudson R, et al. An exercise intervention without weight loss decreases circulating interleukin-6 in lean and obese men with and without type 2 diabetes mellitus. Metab 2007;56:332-8.

25. Blumenthal JA, Sherwood A, Gullette EC, et al. Exercise weight loss reduce blood pressure in men with mild hypertension: effects on cardiovascular, metabolic and hemodynamic functioning. Arch Intern Med 2000;160:1947-58.

26. Tuomilehto J, Lindstrom J, Eriksson JG, et al. Prevention of type 2 diabetes mellitus by changes in lifestyle among subjects with impaired glucose tolerance. N Engl J Med 2001:344:1343-50.

27. Janiszewski PM, Ross R. Physical activity in the treatment of obesity: beyond body weight reduction. Appl Physiol Nutr Metab 2007:32:512 22.

28. Church TS, Earnest CP, Skinner JS, et al. Effects of different doses of physical activity on cardiorespiratory fitness among sedentary, overweight or obese postmenopausal women with elevated blood pressure: a randomized controlled trial. JAMA 2007;297:2081-91.

29. Caudwell $P$, Hopkins $M$, King NA, et al. Exercise alone is not enough; weight loss also needs a healthy (Mediterranean) diet. Pub Health Nutr 2009;12:1663-6.

30. King N, Caudwell P, Hopkins M, et al. Dual process action of exercise on appetite control: increase in orexigenic drive but improvement in mealinduced satiety. Am J Clin Nutr 2009;90:921-7. 